\title{
Articles of interest in other scholarly journals
}

\author{
AVOIDABLE AND UNAVOIDABLE VISITS TO THE \\ EMERGENCY DEPARTMENT AMONG PATIENTS \\ WITH ADVANCED CANCER RECEIVING \\ OUTPATIENT PALLIATIVE CARE \\ - Delgado-Guay MO, Kim YJ, Shin SH, et al. J Pain Symptom \\ Manage 4 Aug 2014. \\ Published Online First 15 Aug 2014. \\ doi:10.1016/j.jpainsymman.2014.07.007
}

Using information from 200 randomly selected patients with advanced cancer receiving outpatient palliative care who visited the emergency department (from a pool of nearly 2000), this study investigated the frequency and clinical characteristics of potentially avoidable emergency department visits. Avisit was considered avoidable if the problem could have been managed in the outpatient clinic or by telephone. Of the 200 patient visits, 46 (23\%) emergency department visits were thought to be avoidable. This was less than the hypothesised amount which was over 50\%. Pain was the most common complaint in avoidable and unavoidable visits (36\%). Altered mental status, dyspnoea, fever and bleeding were present in the unavoidable group only. Constipation and running out of analgesics were more frequent in the avoidable group. In a multivariate analysis, avoidable attendance was associated with non-white ethnicity and constipation, whereas unavoidable attendance was associated with emergency department referral from the outpatient oncology or palliative care clinic, and the presence of baseline dyspnoea. The authors suggest that efforts are needed to improve communication with patients and caregivers and increase support between appointments to reduce avoidable emergency department visits. Furthermore, with increased community services and collaboration between services even the unavoidable emergency department visits might be reduced.

\section{INCIDENCE AND RISK FACTORS FOR SUICIDE AND ATTEMPTED SUICIDE FOLLOWING A DIAGNOSIS OF HEMATOLOGICAL MALIGNANCY}

- Hultcrantz M, Svensson T, Derolf AR, et al. Cancer Med 2014. Published Online First 26 Aug 2014. doi:10.1002/cam4.316

In this Swedish population-based study of nearly 50000 patients with haematological malignancies and over 230000 matched controls the incidence and risk factors for suicide and suicide attempt was evaluated. Information was obtained from national registers and medical records. In total, there were 54 suicides and 158 suicide attempts, of which 36 suicides and 100 suicide attempts were in the first 3 years after diagnosis. There was a twofold increased risk of suicide/ suicide attempt during the first 3 years in patients after diagnosis with haematological malignancies compared to matched controls. Of all haematological malignancies, multiple myeloma was associated with the highest risk (over three times the increased risk). Patients with any haematological malignancy and a pre-existing psychiatric disorder were at a very high risk of suicide and suicide attempt (over 23 times the increased risk). In the patients who committed suicide one-fifth were in a palliative phase and nearly half were in remission (receiving no active treatment). The authors suggest that certain high-risk patients may benefit from early detection and preventive measures.

\section{A SYSTEMATIC REVIEW OF THERAPEUTIC INTERVENTIONS TO REDUCE ACUTE AND CHRONIC POSTSURGICAL PAIN AFTER AMPUTATION, THORACOTOMY OR MASTECTOMY \\ - Humble SR, Dalton AJ, Li L. Eur J Pain 2014. Published Online First 4 Aug 2014. doi:10.1002/ejp.567}

In this systematic review of perioperative interventions reducing acute and chronic pain associated with amputation, mastectomy or thoracotomy, 32 randomised controlled trials were included. Gabapentin/ pregabalin reduced the incidence of acute and chronic pain after mastectomy, but gabapentin was ineffective for vascular amputees with pre-existing chronic pain. Venlafaxine decreased pain (including burning and stabbing pain) at 7 days and 6 months after mastectomy. Intravenous and topical local anaesthetics reduced chronic pain after mastectomy, whereas local anaesthetic infiltration was not shown to be effective. Regional analgesia was generally beneficial for chronic symptoms. Ketamine did not reduce the incidence of chronic pain and generally did not help acute pain after amputation or thoracotomy. In one of two trials, epidural ketamine with bupivacaine reduced acute pain but in neither trail was chronic pain improved. Intercostal cryoanalgesia in thoracotomy generally did not reduce the incidence of acute pain, but interestingly it increased the incidence of chronic pain in four of five trials. Total intravenous anaesthesia reduced post-thoracotomy pain in one study. High-dose remifentanil (with a postoperative epidural) did not influence acute pain, but exacerbated chronic pain versus low-dose remifentanil in a post-thoracotomy study. The authors recommend using this evidence to form a standard approach to patients using drugs to target neuropathic pain in the perioperative management for amputation, thoracotomy and mastectomy. 
THE SYMPTOM BURDEN OF TREATMENT-NAIVE PATIENTS WITH HEAD AND NECK CANCER

- Hanna EY, Mendoza TR, Rosenthal DI, et al. Cancer 2014. Published Online First 4 Nov 2014. doi:10.1002/cncr.29097 In this retrospective study of 748 patients with head and neck cancer who have not received treatment, symptoms, quality of life and health status were investigated using the MD Anderson Symptom Inventory, health-related quality of life and functional well-being scales. One-third of patients had a high symptom burden, with pain, fatigue, distress and disturbed sleep being the most severe symptoms. Pain, fatigue, distress and disturbed sleep at moderate to severe levels (5 or more on the 0-10 scale) were each present in about one-third of patients, with severe levels (7 or more) each being present in around $20 \%$ of patients. Symptom burden was higher in patients with more advanced disease, having a skull base or mucosal tumour and being woman. This study shows the high prevalence of symptoms in treatment-naive patients with head and neck cancer and need to assess patientreported symptoms routinely before treatment. Furthermore, symptom management should be delivered alongside disease directed therapy.

\section{DRUG TREATMENT AT THE END OF LIFE: AN EPIDEMIOLOGICAL STUDY IN NURSING HOMES}

- Jansen K, Schaufel MA, Ruths S. Scand J Prim Health Care 2014;32:187-92.

In a cohort study of 524 patients in three Norwegian nursing homes, diagnoses, drug prescriptions and demographic data were collected from electronic records. On the last day of their life, over $99 \%$ of people were prescribed drug treatments, with $74 \%$ having palliative drugs either alone (27\%) or with curative/preventive drugs (47\%). Of these, $73 \%$ had an anticholinergic, $71 \%$ had morphine, over a half had midazolam and under a half had haloperidol prescribed by injection. Palliative drug prescription was associated with nursing home, length of stay over 16 months and a diagnosis of cancer. Most initiations of palliative drugs and withdrawals of curative/preventive drugs took place on the last day of life. This study indicates that improvements in prescribing at the end-of-life care in nursing homes are needed and imply addressing prognostication and earlier response to palliative needs.

\section{PALLIATIVE CARE AMONG HEART FAILURE} PATIENTS IN PRIMARY CARE: A COMPARISON TO PATIENTS WITH CANCER USING ENGLISH FAMILY PRACTICE DATA

- Gadoud A, Kane E, Macleod U, et al. PLoS ONE 2014;9: e113188.

Using the UK primary care database, Clinical Practice Research Datalink, this study explored if and when patients with advanced chronic heart failure are recognised as needing palliative care and was compared to patients with cancer. This was determined by the patients being entered on the Quality and Outcomes Framework palliative care register in 2009. In this year, over 30000 adults with records on the database had died. Of the useable records (27 689), 5311 (19\%) were on the palliative care register. In total 3122 patients with heart failure died, with 7\% (234) of these being on the palliative care register. This compared to 48\% (3669/7608) of patients with cancer. Furthermore, 29\% (69/234) of patients with heart failure were entered onto the register within a week of their death, compared to $294(8 \%)$ for cancer. This study shows inequity in the timing of and overall recognition of palliative care needs for patients with heart failure compared with cancer in primary care.

\section{DOUBLE-BLIND, PLACEBO-CONTROLLED,} RANDOMISED TRIAL OF OCTREOTIDE IN MALIGNANT BOWEL OBSTRUCTION

- Currow DC, Quinn S, Agar M, et al. J Pain Symptom Manage 2014. Published Online First 14 Nov 2014. doi:10.1016/j.jpainsymman.2014.09.013

In a double-blind multicentre randomised controlled trial in Australia, people with advanced cancer presenting with vomiting secondary to inoperable bowel obstruction, where anticancer therapies were not immediately appropriate, were randomised to placebo or octreotide $(600 \mu \mathrm{g} /$ day $)$ infusion. Both arms received ranitidine, dexamethasone, parenteral hydration and symptomatic therapies. The primary outcome was the number of patient-reported days free of vomiting at 3 days. At 3 days, 87 participants provided data (45 in the octreotide arm); of these 17 (octreotide) and 14 (placebo) were free of vomiting for 3 days. The mean number days free of vomiting were 1.9 (octreotide) and 1.7 (placebo). There was no statistical difference between these groups for the primary outcome. For the secondary outcomes, both groups had a decrease in the mean number of episodes of vomiting between baseline and day 1; the incidence of vomiting over the study period showed a reduced number of episodes of vomiting with octreotide, but this group were twice as likely to be administered hyoscine butylbromide, potentially reflecting increased colicky pain. Octreotide was well tolerated and there was no difference in toxicity between groups. Overall, there was also no difference in nausea, pain or survival between groups. This study does not support the routine use of octreotide in addition to standard care for the reduction of vomiting in inoperable malignant bowel obstruction, however, in view that the secondary end point of episodes of vomiting was reduced with octreotide, the authors recommend further study of octreotide in this setting.

Competing interests None.

Provenance and peer review Not commissioned; internally peer reviewed.

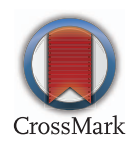

